\title{
Efectividad de Intervención Educativa Para la Aceptación del Suplemento "Vita Niño" en Madres Beneficiarias del Programa PROSPERA
}

\author{
LMC. Luis Ricardo Palmer Serrano, \\ Médico Cirujano, Secretaría de Salud, Tabasco, México \\ Dr. Sergio Quiroz, Gomez, \\ Profesor investigador Universidad Juárez Autónoma de Tabasco, México
}

MCSP. Marisol Guzmán Moreno,

Coordinadora médica Secretaría de Salud, Tabasco, México

URL:http://dx.doi.org/10.19044/esj.2019.v15n33p73

\begin{abstract}
Resumen
El componente salud PROSPERA busca promover la mejor nutrición mediante el suplemento "Vita niño" compuesto de vitaminas y minerales. Sin embargo, a pesar del alto nivel nutritivo, las madres beneficiarias muestran rechazo al uso de éste para otorgárselo a sus hijos y manifiestan desinterés en acudir a las unidades para su provisión. Objetivo: determinar la efectividad de una intervención educativa enfocada al conocimiento, actitud y práctica de madres beneficiarias PROSPERA en el suplemento Vita niño. Material y métodos: estudio cuantitativo, prospectivo y pre- experimental. Se aplicó pretest y un post-test, se evaluó conocimiento, actitud y práctica de las madres, posteriormente se aplicó intervención educativa y por último se evaluó la efectividad de la misma. Resultados: la mayoría de las madres lograron asimilar los conocimientos adecuados sobre el suplemento "Vita niño", la actitud y las prácticas después de la intervención tuvieron un cambio significativo, se observó mejoría en la preparación como en el uso, logrando aprovechar efectivamente los beneficios del producto. Conclusión: la intervención educativa se considera efectiva debido a los cambios significativos, cuando se diseña y ejecuta de acuerdo a las necesidades específicas se logra un impacto real en la población objetivo.
\end{abstract}

Palabras clave: Nutrición, Intervención educativa, Efectividad 


\title{
Effectiveness of Educational Intervention for the Acceptance of the Supplement "Vita Niño" in Mothers Beneficiaries of the PROSPERA Program
}

\author{
LMC. Luis Ricardo Palmer Serrano, \\ Médico Cirujano, Secretaría de Salud, Tabasco, México \\ Dr. Sergio Quiroz, Gomez, \\ Profesor investigador Universidad Juárez Autónoma de Tabasco, México
}

MCSP. Marisol Guzmán Moreno,

Coordinadora médica Secretaría de Salud, Tabasco, México

\begin{abstract}
The health component PROSPERA seeks to promote the best nutrition by means of the supplement "Vita child" composed of vitamins and minerals. However, despite the high level of nutrition, the beneficiary mothers reject the use of nutrition to provide it to their children and show a lack of interest in going to the units to provide it. Objective: determine the effectiveness of an educational intervention focused on the knowledge, attitude and practice of PROSPERA beneficiary mothers in the "Vita child" supplement. Material and methods: quantitative, prospective and pre-experimental study. It was applied pre-test and post-test, knowledge, attitude and practice of the mothers were evaluated, later educational intervention was applied and finally the effectiveness of the same was evaluated. Results: the majority of mothers managed to assimilate the appropriate knowledge about the supplement "Vita niño", the attitude and practices after the intervention changed significantly, improvement was observed in both preparation and use, making effective use of the product's benefits. Conclusion: Educational intervention is considered effective due to significant changes, when it is designed and implemented according to specific needs, a real impact on the target population is achieved.
\end{abstract}

Keywords: Nutrition, Educational intervention, Effectiveness

\section{Introducción}

El estado nutricional de los niños y niñas es un reflejo de su estado general de salud. Cuando los niños y niñas tienen acceso a la provisión suficiente de alimentos, no están expuestos a enfermedades reiteradas y son 
bien cuidados, alcanzan su potencial de crecimiento y se les considera bien alimentados (Encuesta Nacional de Niños, Niñas y Mujeres [ENIM], 2015). En las unidades de primer nivel del estado de Tabasco se entrega a las madres beneficiarias del Componente Salud de PROSPERA Programa de Inclusión Social, el suplemento alimenticio "Vita niño" para los niños de 6 meses de edad hasta cumplir los 59 meses; dicho suplemento fue implementado para atender y prevenir la desnutrición infantil. El sector salud, de acuerdo a la operatividad, está obligado a entregar de manera bimestral los suplementos alimenticios en las unidades de salud y/o a través de los equipos de salud itinerantes (Reglas de operación PROSPERA, 2018).

Los suplementos alimenticios en el sector salud se destinan a niños menores de 5 años y mujeres embarazadas de municipios donde habitan familias con carencia alimenticia. Estos productos constituyen una fuente nutricional importante, ya que proporciona un aporte de energía, proteína, vitaminas "A", "E", "C", "B2", "B12" y ácido fólico necesarios para el desarrollo humano (Estrategia Integral de Atención a la Nutrición [EsIAN], 2018). Con esta estrategia, el componente salud de PROSPERA busca promover la mejor nutrición de la población beneficiaria, en especial para atender y prevenir la mala nutrición en los menores de edad (Reglas de operación PROSPERA, 2018).

El suplemento "Vita niño" está compuesto de vitaminas y minerales en polvo destinado para niños y niñas de los 6 meses a los 5 años de edad. Este suplemento debe mezclarse en una pequeña porción de alimentos semisólidos que el niño o niña pueda comer en el momento (EsIAN, 2018). Sin embargo, de acuerdo con la evidencia local otorgada por el personal operativo, a pesar del alto nivel nutritivo que representa el "Vita niño", las madres beneficiarias del Programa PROSPERA muestran rechazo al uso de éste para otorgárselo a sus hijos y manifiestan desinterés en acudir a las unidades para su provisión.

En México se presentan simultáneamente en la población infantil dos grandes enfermedades que impactan directamente en la vida de los niños y niñas, la desnutrición y sobrepeso-obesidad, son grandes problemas de salud pública que repercuten en el crecimiento y desarrollo de los menores. Desde la perspectiva de la salud, un niño que desde el embarazo de la madre tiene un grado de desnutrición eleva su morbilidad y mortalidad, tanto materna como fetal, ya que si la madre sufre de desnutrición el feto tendrá baja talla y peso, lo cual repercutirá en su desarrollo infantil y a la vez en el desarrollo cognitivo y escolar, creando grandes problemas para su desempeño. La desnutrición tiene grandes orígenes, los cuales pueden estar determinamos por factores biológicos, socioeconómicos y culturales, de donde se destaca importantemente, una inadecuada alimentación ocasionada por ineficientes intervenciones de educación para la salud (Velasco, 2016). 
Por otro lado, los problemas del sobrepeso y obesidad a nivel mundial son factores determinantes para la salud tanto de niños como adultos, dichos estados representan el quinto factor de riesgo de muerte en el planeta, donde México y Estados Unidos tienen el mayor índice. Las principales causas descritas del sobrepeso y la obesidad han sido, el aumento en el consumo de alimentos hipercalóricos, ricos en grasas, sal y azúcar y pobres en vitaminas, minerales y fibra; así como el descenso en la actividad física originado por los modelos de urbanización y sedentarismo de la población. Con esto se observa que el sobrepeso y la obesidad son un problema prevalente de escala mundial (Sama, Amaya y Cuevas, 2015).

La desnutrición en el desarrollo infantil es un problema importante por los efectos adversos sobre la salud y el desarrollo de capacidades. A nivel mundial, casi uno de cada cuatro niños menores de 5 años la padece (165 millones, o el 26\%) (UNICEF, 2017), y en México 1, 100000 (1.9\%) de niños menores de 5 años sufrían de desnutrición crónica (ENSANUT, 2016).

La mortalidad y morbilidad de la madre y el producto también se incrementan por causa de la desnutrición, si la madre la padece durante la gestación el producto será de bajo peso y talla baja y esto repercutirá directamente en el desarrollo del menor (ENSANUT, 2016).

En el estado de Tabasco, la desnutrición representa un significativo problema de salud pública, situándose desde hace varios años dentro de las entidades a nivel nacional con mayor proporción en alguna clasificación de inseguridad alimentaria, de acuerdo con datos de la Encuesta Nacional de Salud y Nutrición, 8 de cada 10 familias en 2012 se encontraban en inseguridad alimentaria (ENSANUT, 2012). Posteriormente, hubo un descenso de la desnutrición primaria de un $38.6 \%$ a un $13.4 \%$ (ENSANUT 2016), sin embargo, no se ha logrado controlar totalmente ni erradicar, puesto que la mal nutrición es una entidad nosológica que afecta a un grupo de población grande de consecuencias mediatas e inmediatas (ESIAN, 2017). Consecuencias que repercuten importantemente en la etapa de 0 a 5 años, donde ocurren los cambios más importantes en el crecimiento y desarrollo; el crecimiento alcanza velocidades elevadas y el desarrollo se caracteriza por el logro de importantes hitos sucesivos en periodos muy cortos de tiempo, es durante esta fase en donde el menor logra su madurez inmunológica y adquiere habilidades y destrezas en su desarrollo psicomotor que lo preparan para su ingreso exitoso al sistema educativo formal (ENSANUT, 2012). En un periodo tan importante para la formación del individuo, la alimentación y la nutrición ocupan un lugar central, al proporcionar la energía y los nutrimentos necesarios para soportar las exigencias del crecimiento y proporcionar las condiciones para que se manifieste un desarrollo ideal.

El suplemento "Vita niño" representa una herramienta importante en el combate a la desnutrición, sus múltiples beneficios ofrecen una mejor 
calidad de vida para el infante, ya que coadyuva al crecimiento y desarrollo óptimo de sus capacidades, al mismo tiempo previene de enfermedades como la obesidad. La falta de conocimiento de todos estos beneficios del suplemento ocasiona que su uso sea limitado o nulo, por ello, las madres del programa PROSPERA deben contar con una correcta información para el uso del suplemento en sus niños, para evitar su desuso y el mal aprovechamiento de este suplemento, ya que esto se traduce en pérdidas económicas y de salud infantil (ESIAN, 2017; IMSS, 2016).

La desnutrición implica tener un peso corporal menor a lo normal para la edad, tener una estatura inferior a la que corresponde a la edad (retraso en el crecimiento), estar peligrosamente delgado o presentar carencia de vitaminas y/o minerales (malnutrición por carencia de micronutrientes o mejor conocida como hambre oculta) (UNICEF, 2007).

Según el "Estado Mundial de la Infancia 2007" de UNICEF, uno de cada cuatro niños y niñas (alrededor de 146 millones) que representa el 27\% de la población de menores de cinco años, tienen peso inferior al normal. Para los niños y niñas cuya situación alimentaria es deficiente, enfermedades comunes de la infancia como la diarrea y las infecciones respiratorias pueden ser fatales. De estos 146 millones, 78 viven en Asia Meridional, 22 en Asia Oriental y el Pacífico, 17 en África Occidental y Central, 16 en África Oriental y Meridional, 8 en Oriente Medio y África del Norte, y en América Latina y el Caribe, 4 millones (UNICEF, 2007).

México, a pesar de haber tenido un notable descenso en los últimos 25 años en algunos tipos de desnutrición, como bajo peso para la talla y bajo peso para la edad, la desnutrición en su forma crónica (baja talla para la edad) persiste como problema de salud pública, existiendo grupos vulnerables para este tipo de desnutrición, como población indígena, las zonas rurales del sur y el quintil de menores condiciones de bienestar (Rivera-Dommarco J., González de Cosío T, Shamah-Levy T, García-Feregrino R., 2013).

De acuerdo a los informes de la última Encuesta Nacional de Salud y Nutrición, para el 2016 la prevalencia de bajo peso para la talla (emaciación), bajo peso para la edad (bajo peso), y baja talla para la edad (desnutrición crónica) fueron de $13.4 \%$. En México la prevalencia de bajo peso en niños menores de 5 años es del $3.9 \%$, mientras que $10 \%$ se clasificaron con baja talla, el $1.9 \%$ de emaciación y un $5.8 \%$ de sobrepeso/ obesidad, así mismo, se conoce que existen deficiencias específicas en algunos otros micronutrimentos, como vitaminas A, C, E, zinc, hierro, ácido fólico, yodo, entre otros. Se estima que los niños desnutridos pierden entre el 12 y $15 \%$ de su potencial intelectual, corren el riesgo de contraer enfermedades infecciosas de 8 a 12 ves más que un niño sano y son más propensos a padecer enfermedades crónicas degenerativas (ENSANUT, 2016). 
Por lo anterior, es de gran importancia que las madres que pertenecen al programa conozcan los beneficios del suplemento y lo otorguen a sus hijos. Hasta el momento no se encuentran registros sobre el uso o desuso del Suplemento alimenticio "Vita niño", solo existe registro del suplemento entregado en las unidades de salud de primer nivel por DICONSA en el estado. El suplemento en el Estado de Tabasco se entrega a 452 madres beneficiarias del Componente Salud del programa PROSPERA Programa de Inclusión Social, que tienen hijos de 6 meses hasta los 59 meses de edad; la situación preocupante en el estado, de acuerdo a las declaraciones del personal operativo y derivado de los reportes entregados en las jurisdicciones sanitarias a la coordinación estatal del componente salud de PROSPERA del estado de Tabasco, no todas las madres aceptan el suplemento (Coordinación estatal PROSPERA, Componente Salud, 2017).

Por lo anterior, se planteó determinar la efectividad de una intervención educativa enfocada al conocimiento, actitud y práctica de madres beneficiarias PROSPERA en el suplemento Vita niño en una localidad de Tabasco México.

\section{Desarrollo}

Entre 1988 y 2016, las prevalencias de las tres formas de desnutrición en menores de cinco años han tenido disminuciones notables. La prevalencia de emaciación (bajo peso para talla) y bajo peso disminuyeron a una cuarta parte de las prevalencias de 1988, para alcanzar valores compatibles con poblaciones sin desnutrición; mientras que la desnutrición crónica (baja talla), aunque disminuyó a la mitad de la prevalencia de 1988, sigue siendo elevada (10 \%), pues representa casi 1 millón 100 mil menores de cinco años en dicha condición (ver figura 1) (ENSANUT 2016).

En las unidades de primer nivel del estado de Tabasco se entrega a las madres beneficiarias del Componente Salud de PROSPERA Programa de Inclusión Social, el suplemento alimenticio para los niños de 6 meses hasta de cumplir los 59 meses, que sirve para atender y prevenir la desnutrición infantil. El sector salud entrega de manera bimestral los suplementos en las unidades de salud y/o a través de los equipos de salud itinerantes.

Con este apoyo que se entrega a la población beneficiaria más vulnerable, el Componente de Salud PROSPERA busca promover la mejor nutrición de la población beneficiaria, en especial para prevenir y atender la mala nutrición (desnutrición y obesidad), a través de la entrega oportuna, dicho suplemento posee vitaminas y minerales en polvo para niños y niñas, necesarios para los requerimientos de su edad. El "Vita niño" se mezcla en una pequeña porción de alimentos semisólidos que el niño o niña puede comer en el momento (ESIAN, 2017). 


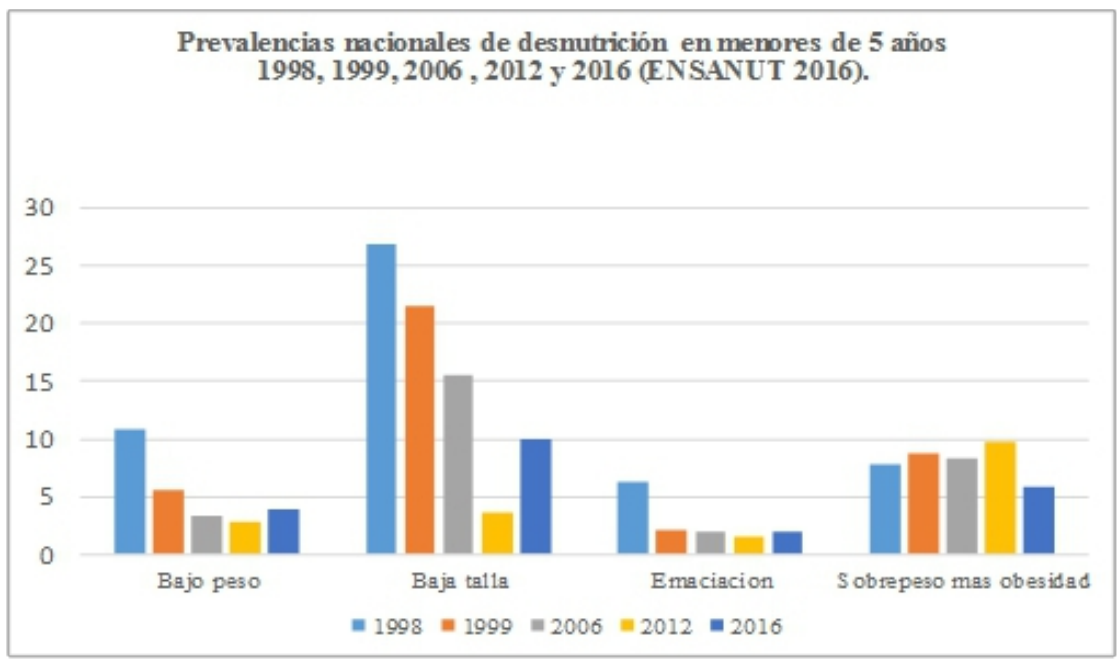

La presente investigación estuvo enfocada en los Conocimientos, Actitudes y Prácticas (CAP) donde se procuró lograr un conocimiento acabado del público objetivo, en este caso las madres de familia beneficiarias con el programa, por ello, como primer momento se inició con un focus group, para explorar el tema y conocer de cerca la problemática expuesta, posteriormente en base a esa información se procedió a construir un instrumento, seleccionar una muestra y realizar la investigación. El estudio es cuantitativo, prospectivo y pre- experimental.

Es prospectivo porque los datos se obtuvieron a partir de un cuestionario aplicado directamente a la población del estudio.

Es pre- experimental porque se aplicó un pre-test y un post-test en un solo grupo, donde se evaluó el conocimiento, actitud y práctica de las madres de niños con un instrumento sobre el suplemento "Vita niño" (pre-test), luego se aplicó la Intervención Educativa y por último se evaluó la efectividad de éste mediante la aplicación del mismo cuestionario (post-test).

La población a estudiar son las madres beneficiarias del Componente Salud de PROSPERA Programa de Inclusión Social con hijos de 6 meses a 59 meses de edad, en la unidad de primer nivel de José María Morelos en Teapa, Tabasco.

La técnica utilizada fue la encuesta, como instrumento se utilizó el cuestionario enfocado a conocimientos, actitud y prácticas de las madres beneficiarias, dicho instrumento fue construido bajo el objetivo de intervención educativa y la prevención de la malnutrición en menores, se procuró en todo momento un acercamiento a las participantes para una comunicación efectiva, respetando su individualidad, utilizando lenguaje sencillo y claro. 
La construcción del instrumento se llevó a cabo mediante 2 fases: una fase cualitativa y una cuantitativa. En la cualitativa, la cual corresponde a la creación del instrumento y validez de contenido, se dividió en 3 momentos: el acercamiento a la población, el juicio de expertos y la revisión del conocimiento disponible. Para ello se realizó en un primer momento, un focus group para la exploración de la problemáticas y las posibles respuestas que se pretendían encontrar (validez de respuesta), en segundo lugar se tuvo un juicio de 4 expertos para la adecuación de ítems de acuerdo a la experiencia de los mismos, una vez construido el instrumento con su lista de ítems, donde se revisaron relevancia, coherencia, suficiencia y claridad con la que han sido redactados los ítems, por último se hizo revisión del conocimiento disponible (validez racional) para asegurar la representatividad de los ítems, aquí los constructos estuvieron mejor definidos, porque se dispuso de literatura especializada en el tema, por lo tanto, no fueron provisionales ni vagos. Se concluyó con 21 reactivos con opción politómica (Osinski y Sanchez, 1998; Garcia, Aguilera y Castillo, 2011).

Dentro de la fase cuantitativa, se evaluaron sus propiedades métricas, como primer paso se evaluó la consistencia interna implicando conocer qué proporción de la variabilidad que tienen los resultados se debe realmente a la variabilidad de la población y no a la del instrumento, para ello se aplicó alfa de Cronbach para su confiabilidad, se realizó una prueba piloto con una muestra de 18 madres, con características similares a la del estudio, cuyos resultados fueron evaluados por la fórmula donde $(\alpha>0,60)$, dando como resultado 0,71 afirmando que la confiabilidad es aceptable.

Para el proceso del análisis de la información se utilizó el paquete estadístico SPSS versión 23. Donde se identificaron cada una de las dimensiones involucradas en el estudio y sus comportamientos respectivos en la pre intervención y post intervención.

El autor manifiesta que no hay conflictos de interés al redactar dicha información, esto se obtuvo mediante permiso tanto de las personas encuestadas, así como de los responsables del Componente Salud de PROSPERA Programa de Inclusión Social del estado de Tabasco, todo ello previo consentimiento informado y exposición de motivos, asegurando la comprensión de los mismos dentro de la población de madres en estudio.

\section{Resultados:}

\section{Características sociodemográficas}

En los indicadores sociodemográficos de las madres participantes, se encontró que, de acuerdo a su grupo etario, un $10.6 \%$ (10) de las madres tenían entre 20 y 25 años de edad, el $46.3 \%$ (74) entre 26 y 30 años, de 31 a 35 años $30.6 \%$ (49), el $11.3 \%$ (18) de 36 a 40 años y una minoría del $1.3 \%$ (2) en el rango de edad de 41 a 45 (ver tabla 1 ). 
Tabla 1. Edad de la madre

\begin{tabular}{cccc} 
& & $\boldsymbol{f}$ & $\boldsymbol{\%}$ \\
\hline $20-25$ & 2 & 17 & 10.6 \\
$26-30$ & 3 & 74 & 46.3 \\
$31-35$ & 4 & 49 & 30.6 \\
$36-40$ & 5 & 18 & 11.3 \\
$41-45$ & 6 & 2 & 1.3 \\
\hline \multicolumn{5}{r}{ Total } & $\mathbf{1 6 0}$ & $\mathbf{1 0 0 . 0}$ \\
\hline
\end{tabular}

En el nivel de estudio de las madres, se observó que el 32.5\% (52) de las participantes tenían el nivel medio superior completo, siendo este el de mayor proporción en las encuestadas, seguido por el 25\% (40) con educación medio superior incompleta, $21.9 \%$ (35) con secundaria completa, el 13.1\% (21) con secundaria incompleta, el 3.8\% (6) con educación universitaria, y un mínimo porcentaje contaba con primaria incompleta, completa y nivel superior terminado con el $1.3 \%$ (ver tabla 2).

De acuerdo con el número de hijos, se pudo identificar que el $53.8 \%$ (86) de las encuestadas contaban con 2 hijos, en igualdad de proporciones con el $21.3 \%$ (34) se encontraban madres con 1 y 3 hijos, la minoría con el $3.8 \%$ (6) declaró tener 4 hijos (ver tabla 3).

Tabla2. Nivel de estudios de la madre

\begin{tabular}{lcc}
\hline a) Primaria incompleta & 2 & 1.3 \\
b) Primaria completa & 2 & 1.3 \\
$\begin{array}{l}\text { c) Secundaria } \\
\text { incompleta }\end{array}$ & 21 & 13.1 \\
d) Secundaria completa & 35 & 21.9 \\
$\begin{array}{l}\text { e) Medio superior } \\
\text { incompleto }\end{array}$ & 40 & 25.0 \\
$\begin{array}{l}\text { f) Medio superior } \\
\text { completo }\end{array}$ & & \\
g) Superior incompleto & 52 & 32.5 \\
h) Superior completo & 6 & 3.8 \\
$\quad$ Total & 2 & 1.3 \\
& $\mathbf{1 6 0}$ & $\mathbf{1 0 0 . 0}$ \\
\hline
\end{tabular}

Tabla 3. Número de hijos

\begin{tabular}{ccc} 
& $\boldsymbol{f}$ & $\boldsymbol{\%}$ \\
\hline 1 & 34 & 21.3 \\
2 & 86 & 53.8 \\
3 & 34 & 21.3 \\
4 & 6 & 3.8 \\
Total & $\mathbf{1 6 0}$ & $\mathbf{1 0 0 . 0}$ \\
\hline
\end{tabular}


En el ámbito de los diferentes tipos de ocupaciones, un gran porcentaje entre las encuestadas declararon ser amas de casa representando el $93.1 \%$ (149), y en una pequeña minoría las dependientes o independientes económicamente con un 5\% y $1.9 \%$ respectivamente, observando con esto que las encuestadas en su gran mayoría se dedican a labores del hogar (ver tabla $4)$.

Por último, dentro de las variables sociodemográficas, específicamente en servicios básicos, una proporción mayoritaria de $67.5 \%$ (108) declararon contar con los servicios de agua, luz y drenaje y en contraparte, un $32.5 \%$ (52) sólo contaban con los servicios de agua y luz.

Tabla 4. Ocupación

\begin{tabular}{ccc} 
& $\boldsymbol{f}$ & $\boldsymbol{\%}$ \\
\hline a) Dependiente & 8 & 5.0 \\
b) Independiente & 3 & 1.9 \\
c) Ama de casa & 149 & 93.1 \\
\hline Total & $\mathbf{1 6 0}$ & $\mathbf{1 0 0 . 0}$ \\
\hline
\end{tabular}

Tabla 5. Servicios básicos

\begin{tabular}{ccc} 
& $\boldsymbol{f}$ & \% \\
\hline $\begin{array}{c}\text { Agua y luz } \\
\text { Agua, luz y } \\
\text { drenaje }\end{array}$ & 52 & 32.5 \\
\hline Total & 108 & 67.5 \\
\hline
\end{tabular}

En la gráfica 1 se observa que, de acuerdo con el estudio, las madres tenían poco conocimiento (25.9\%) sobre el suplemento vita niño, ya que desconocían la información que se debe manejar sobre la nutrición y desnutrición en los niños, para que los niños no tengan problemas de desarrollo, como a su vez era poco su conocimiento sobre el suplemento vita niño. En la misma gráfica se observa que mediante la intervención realizada con las madres en la unidad de salud, existe una diferencia significativa en el conocimiento (75.6\%) de las madres sobre la desnutrición y el suplemento vita niño, evidenciando que adquirieron conocimiento post intervención y fue asimilada por las mismas $(\mathrm{p}<0.05)$. 
Gráfico 1. Conocimiento

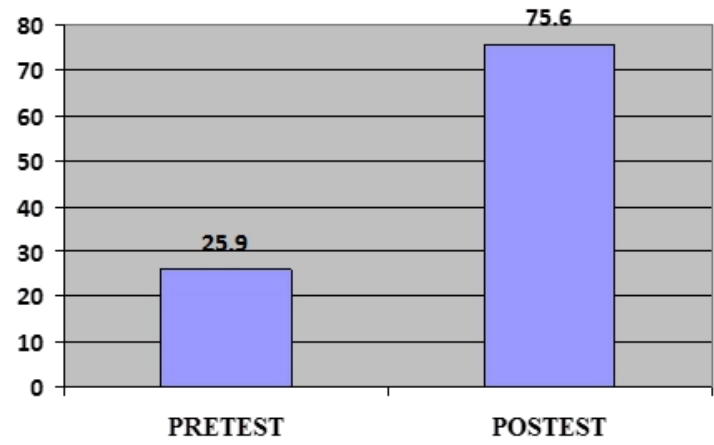

Gráfico 2. Actitud

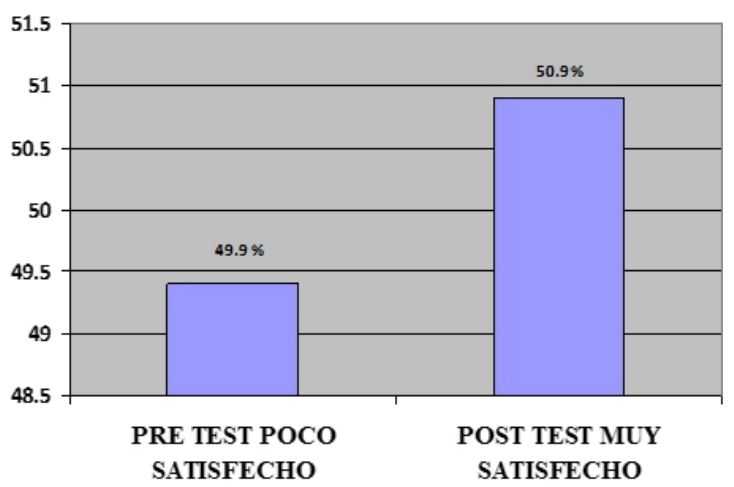

Se evaluó la actitud de las encuestadas observando que en la satisfacción con el suplemento vita niño se encontraban poco satisfechas (49.9\%), con la satisfacción con el suministro del suplemento vita niño con un $48.8 \%$ de poco satisfechas, al evaluar la satisfacción de cómo preparar el suplemento se encontraron poco satisfechas con un $49.4 \%$ y en el grado de satisfacción con la orientación que brinda el personal de salud sobre el suplemento vita niño se observó un 59.4\% de poca satisfacción (ver gráfico 2).

Al realizar la evaluación postest se encontró que las personas encuestadas cambiaron su actitud ya que de estar en el rango de poco satisfechas pasaron a un mayor porcentaje de muy satisfechas encontrando este suceso en los cuatro rangos de satisfacción que se utilizaron, satisfacción con el suplemento vita niño, satisfacción con el suministro del suplemento vita niño, satisfacción de cómo preparar el suplemento vita niño y el grado de satisfacción con la orientación que brinda el personal de salud sobre el suplemento vita niño fue de $50.9 \%, 50.9 \%, 52.1 \%$ y $77.3 \%$ respectivamente. 
Gráfico 3. Práctica

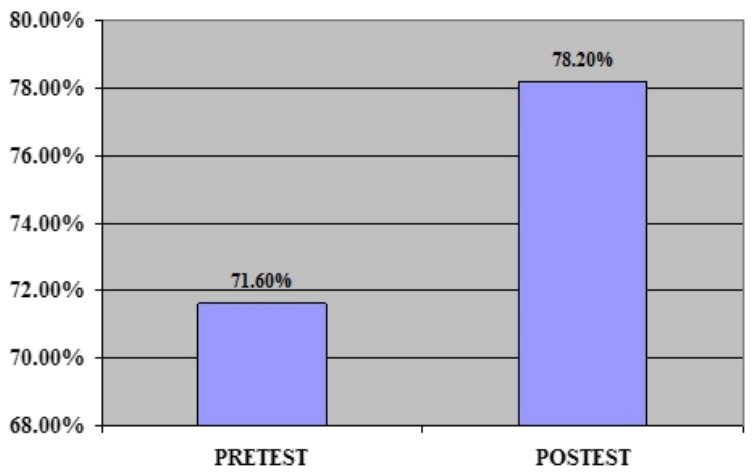

En la gráfica 3, se observa como en las personas que participaron en este estudio se logró una mejora en la práctica de cómo utilizar el suplemento vita niño ya que de estar en un $71.6 \%$, mejoraron a un $78.2 \%$ después de la intervención educativa, sobre la práctica de cómo utilizar el suplemento vita niño en sus hogares.

\section{Discusión}

En un estudio realizado por Zarco y col. (2006) en comunidades beneficiarias con el programa antecesor llamado "Oportunidades" en diferentes zonas del país, se identificó que las madres asociaban el suplemento con leche en polvo, el conocimiento limitado en el producto hacía que la dosificación del mismo no fuera óptima y por lo tanto no otorgara los beneficios necesarios a los menores. Caso similar a lo encontrado en esta investigación, donde las madres beneficiarias a causa de su conocimiento poco claro sobre el Vita niño, proporcionaban de manera incorrecta el suplemento no siguiendo las indicaciones normativas por el personal de salud, así como su aceptabilidad fue en aumento posterior a la intervención educativa destacando entonces que el conocimiento oportuno es un eje primordial para el buen funcionamiento del producto y el aprovechamiento de este.

Una intervención educativa tiene excelentes resultados en una población donde se han detectado deficiencias en conocimiento sobre aquello que se está estudiando, como en el ejemplo de Amaya y col. (2016), los cuales realizaron un estudio del impacto de una intervención educativa, similar al presente estudio se ve de la misma manera que al momento de realizar una intervención educativa en un población la cual tiene el suplemento Vitaniño y no conoce sus beneficios no logran obtener todos los beneficios los cuales no brinda este producto, así mismo pueden conocer los beneficios pero de la misma manera no conocen la manera adecuada en la cual se debe de dar a los niños, es aquí donde nuestra intervención educativa tiene su impacto; así como 
también lo concluyen Chapman y col. (2016) en su estudio de conocimiento en una intervención educativa.

Tal y como se observa en el estudio de Cuevas-Nasu L y col. (2018), realizado a menores de 5 años con bajo peso, emaciación, desnutrición crónica y sobrepeso, en el ámbito nacional en regiones urbanas y rurales donde concluyeron que la desnutrición crónica se encuentra a la baja, pero persisten prevalencias elevadas en grupos vulnerables a la vez que el sobrepeso presento una disminución inesperada, en donde el mayor descenso ocurre entre 1999 y 2006 donde se implementó el programa PROGRESA (ENSANUT 2018), y en conjunto con los programas SOLIDARIDAD, OPORTUNIDADES y PROSPERA se logró descender esta tasa, teniendo bajos niveles de desnutrición crónica y emaciación, pero aún no se logra erradicar del todo teniendo como principal problema el desconocimiento de los beneficios que brinda el suplemento Vitaniño a los menores de 5 años así como también se desconoce en algunas localidades del estado de Tabasco el modo adecuado de preparación.

En el estudio adherencia al consumo de los suplementos alimenticios del programa de Prospera en la reducción de la prevalencia de anemia en niños menores de tres años en el estado de San Luis Potosí, México, Vizuet, Shamh, Goana, Cuevas, \& Méndez (2016) mencionan que la anemia es considerada un factor de riesgo en los niños, que como resultado tiene alteraciones graves durante el crecimiento y el desarrollo, para lo cual se han implementado programas de ayuda alimentaria con la finalidad de prevenir y controlar estas consecuencias. Demostrando que la adherencia al suplemento reduce el riesgo de presentar alteraciones como la anemia, y el programa de Prospera ha contribuido a la disminución de las prevalencias de anemia, y recomiendan llevar a cabo estrategias de mejora para la adherencia al consumo de suplementos alimenticios, es por ello que la intervención educativa que se llevo a cabo en este estudio mejoró a través del conocimiento de las madres las prácticas del consumo del suplemento alimenticio.

Para Menor, Aguilar, Mur, \& Santana (2017) en el estudio realizado mencionan que las intervenciones educativas están dirigidas a fomentar los hábitos de vida saludables, y esto como consecuencia previene las enfermedades y mejora la calidad de vida, considerando un pilar fundamental para la atención de la salud. La finalidad de una intervención educativa es informar y motivar a la población para adoptar y mantener prácticas saludables, propiciando cambios cuando son aplicadas, porque apoyan a la adopción y mantenimiento de estilos de vida saludables, que favorecen la salud, y se obtiene mejores resultados cuando las intervenciones son más duraderas, y son utilides también cuando se trata de enfermedades crónicas. Viendo reflejado dicho estos resultados en el trabajo de investigación, donde 
mejoraron los conocimientos de las madres de menores de cinco años en las prácticas del uso de suplemento alimenticio.

\section{Conclusión}

La implementación de una intervención educativa sobre el uso del suplemento Vita niño en las familias beneficiarias Prospera de la localidad de Morelos en Teapa mejoró las prácticas del uso del suplemento alimenticio, por lo tanto, la intervención educativa se considera que fue efectiva, esto porque se logró un incremento de los conocimientos de las madres de los menores de cinco años.

Los resultados iniciales mostraron que las madres de los menores de cinco años tenían poco conocimiento sobre el suplemento Vita niño, desconociendo la información de la manera en que se debe manejar la nutrición y desnutrición de los niños, así como los problemas de desarrollo que pueden presentar, pero aquellas donde se llevo a cabo la intervención se observó una diferencia significativa, las madres de los menores de cinco años incrementaron sus conocimientos sobre las consecuencias que resultan de la desnutrición y el suplemento Vita niño. Concluyendo que la mayoría de las madres lograron asimilar los conocimientos sobre el suplemento Vita niño, de igual forma la actitud y las prácticas después de la intervención educativa, finalmente la implementación de intervenciones educativas busca solucionar problemas de salud que afectan a la población, y se consideran un complemento primordial para mejorar los estilos de vida en la población, y representan una estrategia en la prevención y control de las enfermedades.

No basta con tener alimento y/o suplementos alimenticios disponible y accesibles, hay que tener idea de cómo prepararlo y saber aprovecharlo para una adecuada nutrición. Diversos problemas mantienen las tasas de mal nutrición, entre las principales se encuentran las madres beneficiarias del programa las cuales tenían una mala actitud y practica respecto al suplemento "Vita niño" ya que no aprovechaban los beneficios de este, debido a que no lo utilizaban adecuada o simplemente no lo utilizaban a pesar de tener el privilegio de contar con este apoyo. Una vez realizada la intervención se observó un cambio significativo en las beneficiarias, en cuanto a su actitud, pensar y comportamientos de acuerdo con el uso del suplemento, se observó mejoría del mismo tanto en la preparación como el uso, logrando aprovechar más efectivamente los beneficios implícitos en el producto. Por lo anterior se concluye que la intervención educativa basada en una cercanía con la población y el análisis de sus características específicas, permite la efectividad del producto y por lo tanto del programa mismo, pudiendo avanzar en los resultados esperados para la población objetivo. 


\section{References:}

1. Amaya M, Arista Y, Díaz J, Paredes S., (2015). Impacto de intervenciones educativas sobre el estado nutricional en preescolares. sector Wichanzao - Trujillo. Recuperado de: file:///C:/Users/Palmer/Downloads/DialnetImpactoDeIntervencionesEducativasSobreElEstadoNutr-6181475.pdf

2. Bovechio A., González W., (2014). Manual para promotores de salud. Estrategia Integral de Atención a la Nutrición (EsIAN) para población beneficiaria de PROSPERA Programa de Inclusión Social.

3. Carta de OTAWA. Organización Mundial de Salud (OMS), (1986). Recuperado http://www1.paho.org/spanish/hpp/ottawachartersp.pdf?ua=1

4. CONEVAL Consejo Nacional de Evaluación de la Política de Desarrollo Social. (CONEVAL), Diagnóstico sobre alimentación y nutrición. (2014), Recuperado de: https://www.coneval.org.mx/Evaluacion/ECNCH/Documents/Diagno stico_sobre_alimentacion_y_nutricion_270715.pdf

5. Cuevas-Nasu et-al, (2018), Tendencias de la mala nutrición en menores de cinco años en México, 1988-2016: análisis de cinco encuestas nacionales. Salud Publica Mex; 60:283-290.

6. Encuesta Nacional de Salud Y Nutrición de México (ENSANUT), (2006).

Recuperado de: https://ensanut.insp.mx/informes/ensanut2006.pdf

7. Encuesta Nacional de Salud Y Nutrición de México (ENSANUT), (2012)

Recuperadode: https://ensanut.insp.mx/informes/ENSANUT2012ResultadosNaciona les.pdf

8. Encuesta Nacional de Salud Y Nutrición de México (ENSANUT), (2016)

\section{Recuperado}

de: https://ensanut.insp.mx/ensanut2016/index.php

9. Fondo de las Naciones Unidas para la Infancia (UNICEF). Diciembre de 2006. Estado mundial de la infancia (2006). Recuperado de: https://www.unicef.org/spanish/sowc07/docs/sowc07_sp.pdf

10. Fondo de las Naciones Unidas para la Infancia (UNICEF). Estimaciones conjuntas de desnutrición infantil del grupo del Banco Mundial, Hallazgos clave. (2015). Recuperado de: https://www.unicef.org/spanish/nutrition/, https://www.unicef.org/media/files/JME_2015_edition_Sept_2015.pd $\mathrm{f}$

11. González, Escobar, González, Shamah, Rivera, (2014). La lactancia materna exclusiva en menores de seis meses se asocia con un mejor 
peso para la longitud en hogares con inseguridad alimentaria en México. Salud Publica Mex;56 supl 1:S31-S38.

12. Instituto Nacional de Salud Pública y UNICEF México. (2016). Encuesta Nacional de Niños, Niñas y Mujeres 2015 - Encuesta de Indicadores Múltiples por Conglomerados 2015, Informe Final. Ciudad de México, México: Instituto Nacional de Salud Pública y UNICEF México. Recuperado de: https://www.unicef.org.mx/Informe2017/Informe-Anual-2017.pdf

13. Kuri, (2018). Promoción de la Salud y Determinantes Sociales 20132018 , Recuperado http://www.ssch.gob.mx/rendicionCuentas/archivos/Promocion\%20d e\%20la\%20Salud\%20y\%20Determinantes\%20Sociales.pdf

14. La Desnutrición Infantil. Causas, consecuencias y estrategias para su prevención y tratamiento. UNICEF (2011). Recuperado de: https://www.unicef.es/sites/unicef.es/files/Dossierdesnutricion.pdf

15. Naciones Unidas (2018). La Agenda 2030 y los Objetivos de Desarrollo Sostenible: una oportunidad para América Latina y el Caribe (LC/G.2681-P/Rev.3), Santiago. Oficina Regional para las Américas de la Organización Mundial de la Salud. Recuperado de: https://www.paho.org/hq/index.php?option=com_content\&view=arti cle\&id=10493: educacion-inocuidad-alimentos-investigacionconocimientos-actitudes-practicas-cap\&Itemid=41279\&lang=es

16. Organización Mundial de Salud OMS (2016). ¿Qué es la malnutrición? Recuperado de: https://www.who.int/features/qa/malnutrition/es/

17. Osinski y Sanchez, (1998). CATEGORÍAS DE RESPUESTA EN ESCALAS TIPO LIKERT. Psicothema. Vol. 10, no 3, pp. 623-631 ISSN $0214 \quad$ - $9915 . \quad$ Recuperado de: http://www.psicothema.es/pdf/191.pdf

18. Reglas de operación PROSPERA 2018. Recuperado de: https://dof.gob.mx/nota_detalle.php?codigo=5509738\&fecha=29/12/ 2017

19. Revista Americana de nutrición clínica, Volumen 100, numero 6, (2014), paginas 1611S-1612S. recuperado de: https://academic.oup.com/ajcn/issue/100/6

20. Rivera, Cuevas, González, Shamah, García. (2013). Desnutrición crónica en México en el último cuarto de siglo: análisis de cuatro encuestas nacionales. Salud Pública de México, 55(Supl. 2), S161S169. http://www.scielo.org.mx/scielo.php?script=sci_arttext\&pid=S003636342013000800013\&lng=es\&tlng=es. 
21. Shamah, Amaya, Cuevas, (2015). Desnutrición y obesidad: doble carga en México. | Vol. 16 | Núm. 5 | ISSN 1607 - 6079. Recuperado de: http://www.revista.unam.mx/vol.16/num5/art34/

22. Velasco, (2016). La desnutrición y la obesidad: dos problemas de salud que coexisten en México. Rev Mex Pediatrica; 83(1):5-6. . Recuperado de: https://www.medigraphic.com/pdfs/pediat/sp-2016/sp161b.pdf

23. Zarco, Mora, Pelcastre, Flores, Bronfman, (2006). Aceptabilidad de los suplementos alimenticios del programa Oportunidades. Salud Publica Mex; 48:325-331. Recuperado de: https://www.redalyc.org/pdf/106/10648407.pdf 